\title{
Hirsh's index and the spirit of capitalism
}

\author{
Dmitry Romanov ${ }^{1, *}$, and Timur Filatov ${ }^{1}$ \\ ${ }^{1}$ Samara State Agricultural Academy, 446442, 2 Uchebnaya str., settlement Ust-Kinelsky, Russia
}

\begin{abstract}
The article presents some data and certain results of the analysis of the validity and effectiveness of modern science-based approaches, methods and specific science-metric criteria. Both positive and negative aspects and trends in science-metric criteria are indicated. A methodological retrospective of the evaluation of scientific work in different time periods is presented. The main attention of the authors is focused on the analysis of the effectiveness of using the Hirsch index to take into account the scientific results and achievements in the field of Russian science. The article contains specific data indicating the complexity and ambiguity of the analyzed situation. The specific examples, figures, facts and analytical data confirm the position of the authors. At the same time, the material of the publication contains quite just wishes and analyses, which, in our opinion, allow, to some extent, to solve (or approach the solution) a difficult task of assessing and taking into account the scientific achievements of contemporary Russian scientists.
\end{abstract}

The purpose of this article is to identify the ideological bases for the spread in the modern Russian Federation of a technology known today for all native scientists to assess the productivity of their activities and the quality of their research as the Hirsch index.

Objective prerequisites for the introduction of such science metrical innovations are quite obvious. In the seventeenth century, when the total number of scientists in Europe (and in the world) did not exceed a thousand people, the total volume of scientific information produced by them was completely accessible to virtually every member of the scientific community, especially as an international science language for all Europeans was Latin. However, already in the nineteenth century, when the total number of scientists reached 100,000 people, not every scientific publication could reach its potential recipient, especially given the fact that scientific works began to be published in national languages. It is enough to recall the founder of genetics, Gregor Mendel, whose research was passed by contemporary biologists. And there are many the same examples in the history of science.

In the middle of the twentieth century, the number of scientists reached 5 million people, having increased in 50 times during 50 years. Since then, it has not grown substantially. However, new information technologies, penetrating, including, in science, have significantly increased the volume of its final conceptual output. Only in the journals indexed in the Scopus system, more than 2 million scientific articles were published in

* Corresponding author: dmitrom@rambler.ru 
2010 [1], which makes it physically impossible not only to read them, but also to simply look through their abstracts on the Internet.

There is a clear overproduction of scientific knowledge, which leads to the paradoxical result, when the most of the articles published by scientists simply do not find their recipients. And we can't say that the proposed works are of poor quality. Just from the huge mass of scientific publications distributed by journals to their potential addressees, it is almost impossible to select something objectively more qualitative than a random sample of average statistics, i.e. with a high probability they are satisfactory articles. Hence the idea of searching for certain objective criteria of quality, which would make it possible to single out the most valuable among millions of works, and to focus the attention of the scientific community on it.

In other words, along with subjective criteria for assessing scientific work by experts who proceed from their personal, purely qualitative ideas about which of the works are "better" and which are "worse," there must be some quantitative criteria for comparing scientific publications that allow to give priority to innovative developments that run counter to the intuition of the community. One of the most ancient ideas of this kind is the famous "Occam's razor" or the principle of simplicity, usually formulated in the form of a maxim: "One should not multiply entities without necessity" [2, p. 6]. In other words, from the two alternative explanations of something, the simplest one is always the most true.

Indeed, scientists have always preferred the simple solutions to more complex and confusing problems. This intuition in the era of the emergence of classical modern science is embodied in the concept of simplicity of nature, which I. Newton expressed in the famous aphorism: "Nature is simple and does not luxuriate with excessive causes of things" [2, p. 38]. Without going into the philosophical analysis of this concept, it can be stated that the evaluation of the quality of the scientist's activity is supposed, first of all, the quantitative evaluation of the final products of the indicated activity, i.e. scientific texts, and not from the point of their content view, but in terms of their form, structural features.

The next step in this direction is the development of various methods of measuring simplicity that would allow each final conceptual structure to give a clear quantitative expression. It is not surprising that the most famous measures of simplicity (N. Goodman, I. Kemeni and others [2, p. 76-137]) are beginning to be actively developed in the second half of the twentieth century, when the tendency to overproduction of scientific information, due to the strengthening of which the science begins to work for the most part idle, becomes quite obvious. It is noteworthy that the active development in this direction was carried out in the domestic philosophy, for example, by A. I. Ujemov, G. N. Povarov, A. Ya. Dubrov, and others [2].

Today we can state that all this colossal work was completed without result, and, not so much for technical, as for ideological reasons. As Goodman pointed out in his time, "There is not a single fundamental objection to the measurability of simplicity, which could not be directed against the measurability of length" [2, p. 80]. The question should be states as follows: Is it necessary for anyone? It is known, for example, that the need for an accounting appeared for people only with the emergence of the institution of private property, when the procedure of counting things acquired a rigid pragmatic meaning. So it is the same with simplicity.

The point is that the quantitative evaluation of finite products of scientific activity, i.e. structural features of the texts, monographs, reports, etc. produced by scientists, implies the regulation of the development of science from the top, i.e. by the communist imperative. The latter makes us recall the notorious totalitarianism, against which the supporters of the most diverse trends of modern Western philosophy actively participated, incl. such prominent postpositivists as K. R. Popper and P. Feyerabend. 
We can consider, that the certain brilliant but unknown scientist writes a brilliant article and sends it to some kind of supernumerary provincial journal. However, the measurement of its structural and textual parameters is of exceptional importance, which automatically initiates the movement: an article that is not known to the author is reprinted by leading national journals, then translated into foreign languages and reprinted in international journals. As they say, the next morning the author wakes up the famous one. At the same time, there is no personal merit in the promotion of his ideas: all this will automatically be done by the corresponding bureaucratic machine, and without his participation.

Another matter is the provincial American scientist Josiah Gibbs, whose story of elevation was told in one of S. D. Haytun's monographs [3, p. 3], which stood at the origins of domestic scientometrics [4]. Being an unknown scientist from the provincial university of the United States, which at that time were not yet a leading scientific power, Gibbs could wait for a long time until his works to be appreciated by the scientific community, if he did not take the process of their advancement into their own hands. He printed 500 copies of his first major monograph, which he sent by mail to the leading European scientists. Only two of them showed interest in this publication, but one of them was D. K. Maxwell, who published the work of Gibbs in Europe, which was the beginning of the future worldwide fame of the provincial American scientist.

In other words, the capitalist imperative presupposes the regulation of science from the bottom, by scholars themselves. The idea of such regulation is very simple: "Do as Gibbs!" In other words, the quality of a scientist's work should be evaluated by the number of citations of his works, since each citation assumes that his scientific ideas not only reached the addressees, but were also appreciated by them. In scientometrics, this approach to assessing the quality of scientific papers is called the "quote-index" method [4, p. 86-121].

More than 30 years ago, when only the "gross" method of counting the number of publications was practiced in the Soviet Union [4], S. D. Hautun pointed to the following principal limitations of this methodology. First, so it is impossible to grasp the vast, any evaluation system covers not all publications, but only the journals that appear in the corresponding sample, compiled taking into account the national and linguistic specifics [4, p. 88]. For example, in such textual bases as Scopus or Web of Science, mainly Englishlanguage publications appear, whereas Russian-speakers are represented to a very small extent. Second, we add from ourselves, monographs cannot get into textual bases, but writing them (for labor) is incomparable with writing articles. At the same time, the value of the monograph, repeatedly quoted in a journal article, coincides with the value of the one-time mentioned article, without any special need attached to the text of the Scopus publication.

We would like, however, to pay attention to another important aspect. The "quoteindex" method essentially identifies the quality of the final conceptual products produced by the researcher, with the degree of its popularity in the relevant scientific community. The latter is quite natural. The mass consumption society assumes not only the "mass culture" that arose as a result of the "mass revolt" described by H. Ortega-and -Gasset [5], but also a kind of "mass science." For example, some famous rapper in the US today is much more popular than Mozart. Hence the poisoned mass culture consciousness concludes: he is much more talented than Mozart. So it is the same to scientists.

The quantitative expression of this idea is the Hirsch index, in our perspective. The practice of its application in modern Russia has already initiated the emergence of a vast array of critical analytical literature. For example, the article by A. D. Polyanin ("Disadvantages of citation indexes and the use of other scientometric indicators" [6]) indicate that "the Hirsch index and its modifications almost completely ignore the most important information about the highly-cited works that scientists are usually proud of. If the Hirsch index analogues were used in sports, the champions would be athletes with 
stable average (but not maximum) results. Conclusion: the Hirsch index is absolutely useless for analyzing the effectiveness of scientific workers, because it can lead not only to gross errors, but also to completely absurd results" [6, p. 136].

This thesis of A. D. Polyanin is illustrated in a small, but very key idea illustrative work of A. M. Nazarenko [7], who, using the scientific electronic library "Elibrary.ru," analyzes the Hirsch index of the leaders of the Russian index of scientific citation by the number of publications. Entering the website of the electronic library and then moving to the author's index, we can easily get a list of the most prolific domestic authors by putting the option "according to the number of publications" in the "sorting" column. Having done these simple operations, we find the most prolific domestic author - Oleg Ivanovich Kvasnenkov, Cand. Tech. Sci., Head of the Technical Department of the All-Russian Scientific Research Institute of Canning and Vegetable Drying Industry [8], which, on December 17, 2017, had 23886 publications and 21390 publications citations (Hirsch index - 56), significantly outstripping the second place with 5194 publications of the famous cardiac surgeon Leo Bockeria.

Website options of "Elibrary.ru" allow to distribute the publications by O.I. Kvasnenkov according to the years. By the way, the most creative fertiliable for him was 2009, when he became the author of 4017 publications, i.e. on average, he published 11 (!) works daily. This supernatural fertility is explained very simply: 23841 , i.e. $99.8 \%$ of O.I. Kvasnenkov's work is a patent. At the same time, $100 \%$ of the publications of the most prolific Russian author were made with co-authors.

In our opinion, the peculiar Achilles heel of the Hirsch index lies precisely in the fact that no distinction is made between the publications of one author and the publications in co-authorship. Both are evaluated in the same way. In other words, if ten authors write one article, each of them will have one publication in the "Elibrary.ru" system; if they simply put their names under each other's texts, they will have 10 publications for each in the indicated system.

As one of the advices given to young scientists in terms of increasing the Hirsch index, the most common is the following: publish in co-authorship with famous scientists. How, however, to distinguish the joint publication of the famous scholar with his student from the publication of the student himself, under which the Master scholar simply put his name in order to promote the work of his student? Obviously, the Hirsch index, in principle, does not allow us to differentiate the above situations.

Either way, the research strategy of O. I. Kvasnenkov proved to be very successful, as evidenced by his last patent fixed in the RSCI system, dedicated to the method of production of frozen tapes [9], which he made together with five co-authors. Continuing the study of M. A. Nazarenko, we grouped the authors of the RPSC according to Hirsch index. Victor Y. Tsvetkov, an adviser of the rector office of the Moscow State Technical University of Radio Engineering, Electronics and Automation (MSTU of REE), Doctor of Technical Sciences and Doctor of Economic Sciences, Professor, the full member of the Russian Academy of Cosmonautics, was the leader with the indicator 98 (as of 17.12.2017). K. E. Tsiolkovsky, the member of the Russian Academy of Natural Sciences, the International Academy of Informatization, the International Academy of Sciences of Eurasia, the Russian Academy of Education Informatization, the prize-winner of the President of the Russian Federation in the field of education, the Honored Worker of Science and Education of RSA, the Honorary Worker of Science and Technology of the Russian Federation, the Honored Worker of Higher Professional Education of the Russian Federation has 1035 publications and 28295 citations [10]. By the way, almost $100 \%$ of the publications of this authoritative author (Elibrary.ru) were written in co-authorship.

Finally, grouping the authors of the RPSC according to the number of citations, as the undisputed leader is Andrey K. Gueim, the Nobel Prize winner in Physics, now a professor 
at Manchester University in the UK. He has "only" 177 publications, and the Hirsch index is 64. At the same time, the number of citations is overvalued - 99572 (as of 17.12.2017). It is understandable! In 2004, Geim, together with his student Konstantin Novoselov, developed a technology for the production of graphene, a new material that is a monatomic carbon layer with unique properties [11]. By the way, Novoselov, the author of 193 publications, takes the second place in the number of citations in the RSCI - 98854 (Hirsch index - 70). And the vast majority of Heim's articles were written in co-authorship.

Let us summarize what has been said. Of course, scientists will adjust to the criteria for evaluating their activities offered by officials, for example, by joined into "mafia" groups to increase the number of publications (in co-authorship) and raising the Hirsch index by meaninglessly citing each other's work using the algorithm used to calculate the Hirsch index. It is noteworthy that almost immediately after the introduction of the relevant evaluation parameter with the mandatory registration of university professors in the RSCI system, Internet publicizes the services for increasing the Hirsch index for the moderate payment.

In other words, the attempt to intensify scientific activity through more stringent control of it once again led to the result, which is directly opposite to the expected one. At all levels of science and education of the Russian Federation, a violent imitation of creativity began. That is, if years ago only the unscrupulous bosses were interested in co-authorship, thus promoting their scientific schools and making their names in science, the new control system devalued everything that is done in the Russian scientific community as a whole (and indeed in the world), putting scientists before a tough dilemma: either write for selfsatisfaction and be eternal outsiders, relics of the outgoing noosphere, or actively fabricate for the most part a meaningless science-like delusion. In co-authorship. And move forward, thus, in a variety of ratings.

If the requirement of "shaft" (the method of counting the number of publications) led to the fact that the authors lost readers, to which it was impossible to reach through tons of this "shaft," then the quotation-index method deprived the readers of the authors. Indeed, who among 5-15 co-authors really wrote this particular text? Or, perhaps, the author remained unknown, because he was not considered necessary to be mentioned in the list of authors, because there simply was not enough place for him? Is not this the realized "death of the author", about which Roland Barth told the mankind half a century ago [12]? However, another definition more suited to the situation we are considering: the "author's murder" in science and education of the Russian Federation by the "quote-index" method.

And in conclusion. On the Internet, we managed to find an article by the pioneer of scientometrics S. D. Khaytun, in which he commented on his departure from the Institute of History of Natural Science and Technology named after S. I. Vavilov of RSA [13]. The scientist writes that he was "not the last (worst) person" in this institute. "Nevertheless, I retired from the Academy of Sciences, where I worked since 1972," he points out. "I will work at home on monographs, live on retirement. It is difficult, of course, but I will be free from the nauseating oppression of the Federal Agency of Scientific Organizations (FASO)" [13]. In particular, the "thinkers" working in FASO come up with all the new indicators of scientific work that do not have any scientific meaning (I speak this responsibly as the author of two monographs on science metrics), and we ourselves must "groom the rope for our necks", counting these figures for ourselves. For example, at first FASO agreed the Hirsch index was one of the citation indicators borrowed from the West, but was not very popular there because of its uselessness. Practice has shown that if you correctly register your publications in the Russian Scientific Citation Index (RSCI), then this indicator can be significantly raised. I, for example, raised my Hirsch (with the help of colleagues) from 8 to 15. What an objective indicator is this! The indicator which so lends itself to "rubbing the thermometer!" [13]. 
In this connection, we would like to make the following concluding observations. First, an objective assessment of the authors, in principle, is impossible, because it is historical. The maximum that can be counted on is a more or less objective evaluation of the products of the activity of these authors, in this case - scientific texts. This kind of assessment can be changed and improved, and, without any harm to the authors themselves.

Second, the art (perfect) of management means the ability of ignoramuses to select intuitively and promote the specialists whose activities should again be evaluated by managers on the basis of final results, primarily in the field of economics, and not according the ability of these specialists to be effectively exposed in various ratings of foreign origin. Otherwise, the financial flows, controlled by these managers will be directed to another meaningless construction of the "Potemkin villages," while the real "villages" would come to the final decline and desolation.

\section{References}

1. M. N. Kotsemir, Dynamics of Russian and world science through the prism of international publications (Moscow, 2012)

2. E. A. Mamchur, N. F. Ovchinnikov, A. I. Ujemov, Principle of simplicity and measures complexity (Nauka, Moscow, 1989)

3. S. D. Khaytun, Problems of post-communism in Russia (AGAR, Moscow, 1996)

4. S. D. Khaytun, Scientometrics: state and prospects (Nauka, Moscow, 1983)

5. J. Ortega y Gasset, The revolt of the masses (AST Publishing House, Moscow, 2017)

6. A. D. Polyanin, Mathematical Modelling and Numerical Methods, 1, 1 (2014)

7. M. A. Nazarenko, International Journal of Applied and Fundamental Research, 6 (2013)

8. The leader of the scientific publications according to the version Elibrary.ru (http://sisv.com/news/2013-08-26-115, 2017)

9. N. E. Kaukhchishvili, T. P. Nitsenko, N. T. Dontsova, N. N. Mashkova, G. A. Belozerov, O. I. Kvasnenkov. Method of production of frozen zrazy: The patent for invention RUS 2625971 11.01.2016. (Moscow, 2016)

10. Prospects of science education (https://pnojournal.wordpress.com/editors/tsvetkov, 2017)

11. A. K. Geim, Phys., 181 (2011)

12. R. Bart, Selected works: semiotics, poetics (Publishing group "Progress," Moscow, 1994)

13. S. Khaytun, I don't want to be a slave to FASO, or why I quit the Institute of the Russian Academy of Sciences (http://www.sib-science.info/ru/ras/ne-khochu-bytrabom-fan-08062016, 2017) 